\title{
Conformal and kinetic couplings as two Jordan frames of the same theory
}

\author{
Conformal and kinetic couplings
}

D. V. Gal'tsov ${ }^{\mathrm{a}}$

Faculty of Physics, Moscow State University, 119899 Moscow, Russia

Received: 5 April 2020 / Accepted: 4 May 2020 / Published online: 19 May 2020

(C) The Author(s) 2020

\begin{abstract}
Certain scalar-tensor (ST) theories with nonminimal coupling of the scalar field to curvature may admit an Einstein frame representation, where gravity is described by the Einstein-Hilbert action plus the scalar sector. Between them, some theories exactly coincide in their respective Einstein frames. If transformations between Jordan and Einstein frames are invertible, these theories can be associated with two Jordan frames of the unique theory. Such successive dualities can connect theories with non-derivative coupling, like $R \phi^{2}$, with derivatively coupled theories, like Horndeski and DHOST. In absence of matter, these are equivalent, though looking very different. We show the existence of a successive duality between the $R \phi^{2}$ theory and a recently found Palatini kinetically coupled theory, which both look in their Einstein frames as Einstein theory minimally coupled to scalar. Transforming singular exact solutions of the latter to Jordan frames, we compare desingularization properties of the above two theories which both violate the null energy condition. It is found that kinetically coupled theory has stronger desingularization features, exhibiting possibility of Genesistype behavior of the homogeneous and isotropic cosmological solutions.
\end{abstract}

\section{Introduction}

Scalar-tensor theories with non-minimal coupling of the scalar field to curvature remain the theories of the first choice in the search of modified gravity which could explain inflation, dark energy and (possibly) dark matter, for recent reviews see Refs. [1-6]. The unusual properties of such theories are closely related to violation of the energy conditions of General Relativity. Recall, that the strong energy condition within the Friedmann-Robertson-Walker (FRW) cos-

a e-mail: galtsov@phys.msu.ru (corresponding author) mology means that the energy density and pressure satisfy the inequality $\epsilon+3 p \geq 0$, implying that the universe is non-accelerating. This condition can be violated already by the minimally coupled scalar field with a potential. The null energy condition (NEC), $\epsilon+p \geq 0$, is more robust; it is violated only in non-minimal ST theories, such as the conformally coupled scalar-tensor theory $[7,8]$ or derivatively coupled theories: Horndeski, beyond Horndeski and degenerate higher order scalar-tensor theories (DHOST) [9]. Violation of NEC can drastically change behavior of the spacetime metrics near static or cosmological singularities both for non-derivative and derivative couplings.

A notable example of a non-derivative ST theory violating NEC is the $\xi R \phi^{2}$ theory, which is conformal (in four dimensions) for $\xi=1 / 6$. Its Jordan frame (which will be called conformal frame in what follows irrespectively to the value of $\xi$ ) is related to the Einstein frame by a conformal transformation, which is invertible excluding singular points of the conformal factor. Since the non-minimal term contains second derivatives of the metric, its effective stress tensor differs from that of the minimal theory. Recall that it was first found by Chernikov and Tagirov [10], then rediscovered in the QFT context as an "impoved energy momentum tensor" by Callan: [11], and further by Parker [12] in curved spacetime (for some later discusstion see, e.g., [13]. That this tensor violates various energy conditions was noted long ago by Beckenstein [14], demonstrating possibility of avoiding the cosmological singularity. Later work in this directions included, in particular, the Refs. [15-18].

This theory also attracted attention in connection with inflation. For $\xi \neq 1 / 6$, the $\xi R \phi^{2}$ theory is no longer conformal, but it turned out to be useful for inflation in the case of large negative $\xi$. In fact, earlier attempts to associate inflation with the only physically known scalar field, Higgs, were not successful in the case of minimal coupling, since the mass needed to accomodate the observed density perturbations had 
to be of the order $10^{13} \mathrm{GeV}$, and the self-coupling constant to be very small, $\lambda \sim 10^{-13}$. This was improved by including the non-minimal coupling of the type $\xi R \phi^{2}$ [19] with large negative $\xi$, in which case the tuning of the Higgs mass could be diminished [20], but at the price of unnaturally large value of $|\xi|$. Somewhat better the situation with perturbations of the $\xi$-Higgs inflation was in the Palatini treatment of the same theory [21]. Still, the problem persisted with unitarity for quantized perturbations [22]. To cure this, the derivatively coupled ST theory was suggested (new Higgs inflation) [23], which was also studied in combination with the $\xi R \phi^{2}$ term [24]. Moreover, it was found that derivatively coupled STs can provide inflationary attractors without scalar field potentials at all [25-32].

Extremely popular became the derivatively coupled ST theories after discovery of the ghost-free massive gravity and the Galileon theories. This led to Horndeski [33] class, rediscovered as generalized Galileons [34,35], beyond-Horndeski $[36,37]$ and DHOST theories encompassing finally the whole set $[4,38]$. Initially they were proposed in the metric formalism, but later also considered in Palatini [39-48] and hybrid $[45,49]$ versions. Generically, Palatini formulation of nonminimal theories leads to equations of motion different from their metric counterpart, however, the question may be subtle in some cases (see discussion of the $f(R)$ theory in Ref. [50]).

Proliferation of derivatively coupled theories led to attempts to explore general properties of the ST landscape [4,51]. An important tool for this is provided by disformal dualities which are typically present in this framework. Introduced by Bekenstein [52] on the basis of Finsler geometry as generalization of conformal transformations, they reappeared in derivatively coupled ST theories as relations between different frames [53-57]. They can be used to obtain new Lagrangians, or as the solution generation tools [44,57], they also naturally arise in Palatini versions of STs $[42,44,48]$ as relating two canonical frames. Special class constitute invertible disformal transformations: these do not change the number of degrees of freedom [58-65], so two ST theories related by an invertible disformal transformation mathematically are equivalent. This is true, of course, only for theories without matter, since the matter makes the choice of the physical frame where the matter enters in a canonical way.

Here we want to draw attention to the group property of invertible transformations, either conformal, or disformal: two successive transformations generate another invertivle transformation up to subtleties with their respective domains (here we will not discuss restrictions due to domain definitions which are certainly important in general). Consequently, two different ST theories, admitting an Einstein frame, in which the metric sector is described by the EinsteinHilbert action, and the scalar sector is the same, will be successively dual to each other. If the scalar sector in the Ein- stein frame is described by equations of the second order, both such STs will be free from Ostrogradsky instabilities. Of particular interest is the class of ST theories which are invertibly reduced in their Einstein frames just to minimal Einstein-scalar theory (MES). Then you can use frame transformations as solution generating technique to explore new theories in the situations which are considered as problematic in the General Relativity, especially near singularities.

Recently, a new type of behavior attracted attention in the cosmological solutions of STs with higher derivatives, such as Galileon [66] and DHOST [67] theories. The univers starts from (or passes through after previous evolution) the Minkowsky space and demonstrates there a sharp violation of NEC, implying that the Hubble parameter satisfies the condition $\dot{H} \gg H^{2}$. In this case, the usual inflation scenario can sometimes be replaced by an alternative scenario called Genesis [66]. It woud be interesting to know whether this behavior can occurs in more familiar ST theories including the non-derivatively coupled ones. Here we address this question using exact solutions which can be generated in the class of MES-dual theories. Mention in passing that the modification of Penrose-Hawking singularity theorems with weakened energy conditions was recently discussed in [6870].

Consider two different STs which reduce to MES in their respective Einstein frames and which, therefore, are successively dual. Both Brans-Dicke and $\xi R \phi^{2}$ theories, as well some other STs non-minimally coupled to scalar without derivatives, share this property, and the transition to their Einstein frame is done through invertible conformal transformations. Using any exact solution of the MES theory, it is possible to generate solutions of these two non-minimal STs in their Jordan frames. Moreover, if the transformations between frames are invertible, one can start with a known Jordan frame solution of one ST, convert it to the Einstein frame, and then convert again into the Jordan frame of another ST. To find such dual pair of theories within the set of derivatively coupled STs is a non-trivial task. Here we discuss one such theory which belongs to kinetically coupled class and which does not belong to the Horndeski class in the metric formalism (neither to DHOST).

Desingularization in the $\xi R \phi^{2}$-theory is well-known. Transformation to the Einstein frame in STs was discussed by Wagoner in 1970 [71] and Bronnikov in 1973 [72] (apart from ealier discussion in the context of Brans-Dicke theory). Bekentstein adressed the theory $R \phi^{2} / 6$ [73] and formulated transformations in an elegant form using the hyperbolic functions. Having applied them to one of the FicherJanis-Newman-Winicour (FJNW) static spherically symmetric solution of MES with a singular "would be" horizon, he obtained (with the Maxwell field added) an asymptotically flat black hole [75] which coincided with the solutions found in 1970 by Bocharova, Bronnikov and Melnikov [74]. Beken- 
stein's duality was independently rediscovered and discussed by many people [76-80]. Conversion to an Einstein frame (but not to MES) was found for non-minimal models including arbitrary functions $F(\phi) R$ and $F(R, \phi)$ [81], including the cosmological constant [82] or potentials [83] in the MES frame, in higher dimensions [84]. Later, the Palatini version of this theory was also discussed, for relationship with the metric approach and the references, see [21].

At the same time, physical (non)equivalence of the Jordan frame and the Einstein frame was subject of long discussion, for a review of papers prior to 1994 see $[83,85,86]$, for more recent aspects and references see [87-93]. Invertible conformal symmetries preserve Noether symmetries of cosmological solutions in two frames [94]. Further interesting aspects of the frame relationship in the cosmological context is related to the concept of conformally connected lagrangians [95]. But two dual forms of scalar-tensor theory differ significantly when matter terms are added to them. Another aspect of (non)equivalence is related to issues of stability and the quantum-level properties, this also remain the subject of discussion [96-99]. All these discussions are relevant to conformally related Jordan and Einstein frames, the case of disformally related frames for the moment is not elaborated in detail.

Here we will explore the difference of two Jordan frames of successively dual theories near the MES-frame singularities, revealing that the derivative coupling ensures stronger violation of NEC than the conformal coupling. Namely, the static singular MES solution becomes a black hole horizon in the $R \phi^{2}$ Jordan frame, but exhibit globally regular behavior in the new kinetic theory frame. The cosmological MES singularity, within the non-derivative ST, becomes just the start of the universe from Minkowsky spacetime with the subsequent decelerating expansion, while in the kinetic frame it exhibits sharp violation of NEC generating the Genesis-type behavior.

The plan of the paper is as follows. In Sect. 2 we revisit the non-derivative $\xi R \phi^{2}$ theory discussing transformations to the Einstein frame, NEC violation and other aspects. In Sect. 3 we consider the two-coupling derivative theory, which for some particular ratio of the couplings reduces to Horndeski class in the metric approach. We then adopt Palatini formulation, showing that the theory is ghost-free for arbitrary couplings while for another ratio of two couplings the theory it is disformally dual to MES and, therefeore, successively dual to the theory $\xi R \phi^{2}$. In Sect 4 we use dualities as generating technique to construct Jordan frame duals for the static FJNW solution and the stiff-matter FRW cosmology in the Jordan frames of both theories, comparing their desingulariziation features. The results are summarized in Sect. 5 .

\section{Non-derivative theory $\xi R \phi^{2}$}

For the reader's convenience, we briefly review the main features of this theory, which is one of the oldest ST with non-minimal non-derivative coupling [73,76-80]:

$S=\int d^{4} x \sqrt{-g}\left(R-g^{\mu \nu} \partial_{\mu} \phi \partial_{\nu} \phi-\xi R \phi^{2}-2 V(\phi)\right)$,

where we set $8 \pi G_{N}=1$. Variation of this action with respect to the metric and the scalar field gives the Euler-Lagrange equations:

$G_{\mu \nu}=T_{\mu \nu}^{\phi}, \quad \square \phi-\xi R \phi=0$,

where the stress energy tensor is

$$
\begin{gathered}
T_{\mu \nu}^{\phi}=\partial_{\mu} \phi \partial_{\nu} \phi-\frac{1}{2} g_{\mu \nu} g^{\alpha \beta} \partial_{\alpha} \phi \partial_{\beta} \phi-g_{\mu \nu} V+ \\
+\xi\left[g_{\mu \nu} \square-\nabla_{\mu} \nabla_{\nu}+G_{\mu \nu}\right] \phi^{2}
\end{gathered}
$$

The Weyl transformation $\phi \rightarrow \Omega^{-1} \phi, g_{\mu \nu} \rightarrow \Omega^{2} g_{\mu \nu}$, leaves the Eqs. (2) invariant if $\xi=1 / 6$. In addtion, $T_{\mu \nu}^{\phi} \rightarrow$ $\Omega^{-2} T_{\mu \nu}^{\phi}$, if $V=0$. Then the trace of $T_{\mu \nu}^{\phi}$ vanishes on shell [10-12]:

$g^{\mu \nu} T_{\mu \nu}^{\phi}=\phi\left(\square \phi-\frac{R}{6} \phi\right)=0$,

and $R=0$, as expected for a conformal field, and so $\square \phi=0$ on shell.

Attributing the Einstein tensor term in (3) to the left hand side of the Einstein equation, we obtain the effective stress tensor:

$$
\begin{aligned}
G_{\mu \nu}= & T_{\mu \nu}^{\mathrm{eff}}=\left(1-\xi \phi^{2}\right)^{-1}\left[\partial_{\mu} \phi \partial_{\nu} \phi-\frac{1}{2} g_{\mu \nu} g^{\alpha \beta} \partial_{\alpha} \phi \partial_{\beta} \phi\right. \\
& \left.-g_{\mu \nu} V+\xi\left(g_{\mu \nu} \square-\nabla_{\mu} \nabla_{\nu}\right) \phi^{2}\right] .
\end{aligned}
$$

\subsection{Einstein frame}

To pass to Einstein frame we recale the metric $[73,77]$

$\hat{g}_{\mu \nu}=\Omega^{2} g_{\mu \nu}, \quad \Omega^{2}=\left|1-\xi \phi^{2}\right|$,

arriving at the following action 
$S=\int d^{4} x \sqrt{-\hat{g}}\left(\hat{R}-F^{2}(\phi) \hat{g}^{\mu \nu} \partial_{\mu} \phi \partial_{\nu} \phi-\hat{V}(\phi)\right)$,

where $\hat{R}$ is the Ricci scalar of the new metric,

$\hat{V}=\frac{V}{\left(1-\xi \phi^{2}\right)^{2}}, \quad F^{2}=\frac{1-\xi(1-6 \xi) \phi^{2}}{\left(1-\xi \phi^{2}\right)^{2}}$,

are the new potential and the kinetic prefactor.

To put the kinetic term into the standard form one has to pass to a new scalar field $\hat{\varphi}$, related to $\phi$ via

$\frac{d \hat{\varphi}}{d \phi}=F(\phi)$

This redifinition results in the Einstein frame action

$S_{\mathrm{E}}=\int d^{4} x \sqrt{-\hat{g}}\left(\hat{R}-\hat{g}^{\mu \nu} \partial_{\mu} \hat{\varphi} \partial_{\nu} \hat{\varphi}-\hat{V}(\hat{\varphi})\right)$,

where the potential has to be expressed through the new scalar field. The Eq. (10) can be integrated explicitly as follows [77]:

$\hat{\varphi}= \begin{cases}\sqrt{\nu / \xi} \arcsin (\sqrt{\nu \xi} \phi)+\sqrt{3 / 2} \ln \left|\frac{W_{+}}{W_{-}}\right| & \xi<1 / 6, \\ \sqrt{3 / 2} \ln \left|\frac{1+\sqrt{6} \xi \phi}{1-\sqrt{6} \xi \phi}\right|, & \xi=1 / 6, \\ \sqrt{\nu / \xi} \operatorname{arcsinh}(\sqrt{\nu \xi} \phi)+\sqrt{3 / 2} \ln \left|\frac{W_{+}}{W_{-}}\right|, & \xi>1 / 6,\end{cases}$
$v=|1-6 \xi|, W_{ \pm}=\sqrt{6} \xi \phi \pm \sqrt{1-\nu \xi \phi^{2}}$.

For $\xi=1 / 6, V=0$ these transformations reduces to the original form of conformal transformation found by Bekenstein [73] and suggested as generating technique to construct solutions $R \phi^{2} / 6$ theory from the solutions of MES: from any solution $\hat{g}_{\mu \nu}, \hat{\varphi}$ of the theory,

$S=\int d^{4} x \sqrt{-\hat{g}}\left(\hat{R}-\hat{g}^{\mu \nu} \partial_{\mu} \hat{\varphi} \partial_{\nu} \hat{\varphi}\right)$

a solution $g_{\mu \nu}, \phi$ to the theory $R \phi^{/} 6$ theory is obtained via the transformation

$g_{\mu \nu}=\left(1-\phi^{2} / 6\right)^{-1} \hat{g}_{\mu \nu}, \quad \phi=\sqrt{6} \tanh (\hat{\varphi} / \sqrt{6})$

This transformation is invertible, provided the value $\phi^{2}=6$ is not reached, an inverse map being

$\hat{g}_{\mu \nu}=\cosh ^{2}(\phi / \sqrt{6}) g_{\mu \nu}, \quad \hat{\varphi}=\sqrt{6}(\tanh )^{-1}(\phi / \sqrt{6})$.

Maeda [81]) had shown that a more general theory with the non-minimal functional coupling $F(R, \phi)$ can be reduced to the Einstein-Hilbert term plus scalar fields (but not MES).

\subsection{Generating Mexican hat potential}

Now let's start with the MES theory with the cosmological constant:

$S_{\mathrm{E}}=\int d^{4} x \sqrt{-\hat{g}}\left(\hat{R}-2 \Lambda-\hat{g}^{\mu \nu} \partial_{\mu} \hat{\varphi} \partial_{\nu} \hat{\varphi}\right)$

and apply the inverse duality transformations (15). The cosmological term then generats in the Jordan frame action a potential term [82]:

$S=\int d^{4} x \sqrt{-g}\left(R-g^{\mu \nu} \partial_{\mu} \phi \partial_{\nu} \phi-2 V-\frac{1}{6} R \phi^{2}\right)$,

which has a Mexican hat shape

$V=\frac{\lambda}{4}\left(\phi^{2}-v^{2}\right)^{2}$

where in dimensionful units

$\lambda=\frac{8 \pi G_{N} \Lambda}{9}, \quad v^{2}=\frac{3}{4 \pi G_{N}}$,

and $G_{N}$ is the Newton constant. Note that the vacuum expectation value $v$ of Higgs is not a free parameter, but up to a factor is equal to the Planck's mass. In particular, one can not set $V=0$, so the resulting theory is not conformal. The case of more general potentials in MES-frame was considered in [83].

\subsection{Violation of NEC}

The null energy condition for the effective stress-tensor reads

$T_{\mu \nu}^{\mathrm{eff}} l^{\mu} l^{\nu} \geq 0, \quad l^{\mu} l_{\mu}=0$

for any null vector $l^{\mu}$. Substituting (6), one obtains [8]:

$\left(1-\xi \phi^{2}\right)^{-1}\left[\left(\phi^{\prime}\right)^{2}-\xi\left(\phi^{2}\right)^{\prime \prime}\right] \geq 0$,

where $\phi_{\mu}=\partial_{\mu} \phi$, and the prime operation is defined as $\phi^{\prime}=l^{\mu} \nabla_{\mu} \phi$. Therefore, for $\xi<0$, any local maximum of $\phi^{2}$ violates NEC, similarly for $\xi>0$, any local minimum of $\phi^{2}$ with $\xi \phi^{2}<1$ and any local maximum of $\phi^{2}$ with $\xi \phi^{2}>1$ violate NEC.

\subsection{Palatini}

In the Palatini (or metric-affine) version [21], connection is treated as independent field which has to be fixed by varying 
the action $S_{P}(\hat{\Gamma}, g)$ :

$$
\begin{aligned}
S_{P}= & \int d^{4} x \sqrt{-g}\left(\hat{R}_{\mu \nu}(\hat{\Gamma}) g^{\mu \nu}\left(1-\xi \phi^{2}\right)\right. \\
& \left.-g^{\mu \nu} \partial_{\mu} \phi \partial_{\nu} \phi-2 V(\phi)\right) .
\end{aligned}
$$

Generically, independent variation of the connection generates non-metricity and torsion. In this case the Ricci tensor is not symmetric. However, the action (22) includes only symmetric part of it. As a result, it is invariant under projective transformation of the connection (for a recent discussion see [100]

$\Gamma_{\mu \nu}^{\lambda} \rightarrow \Gamma_{\mu \nu}+A_{\mu} \delta_{v}^{\lambda}$,

in which case torsion can be consistently set to zero [101103]. Then the Ricci tensor $\hat{R}_{\mu \nu}(\hat{\Gamma})$ should be varied as

$\delta \hat{R}_{\mu \nu}=\hat{\nabla}_{\lambda} \delta \hat{\Gamma}_{\mu \nu}^{\lambda}-\hat{\nabla}_{\nu} \delta \hat{\Gamma}_{\mu \lambda}^{\lambda}$,

where the covariant derivative with respect to the Palatini connection is understood. Variation of (22) with respect to $\hat{\Gamma}$, after integration by parts, leads to the following equation

$\hat{\nabla}_{\lambda}\left[g^{\mu \nu}\left(1-\xi \phi^{2}\right) \sqrt{-g}\right]=0$.

With the field redefinition (7), one can rewrite this as

$$
\hat{\nabla}_{\lambda}\left(\hat{g}^{\mu \nu} \sqrt{-\hat{g}}\right)=0,
$$

showing that the Palatini connection is nothing but the LeviCivita connection of the Einstein frame metric.

Variation of (22) with respect to metric $g_{\mu \nu}$ gives the Einstein equation which can be written in terms of the Einstein frame metric as follows

$$
\begin{aligned}
\hat{R}_{\alpha \beta}\left(\delta_{\mu}^{\alpha} \delta_{\nu}^{\beta}-\frac{1}{2} \hat{g}_{\mu \nu} \hat{g}^{\alpha \beta}\right)= & \frac{\phi_{\mu} \phi_{\nu}}{1-\xi \phi^{2}} \\
& -\frac{1}{2} \hat{g}_{\mu \nu}\left(\phi_{\alpha} \phi_{\beta} \hat{g}^{\alpha \beta}+2 V\right) .
\end{aligned}
$$

\section{Derivative coupling}

\subsection{The metric theory}

Consider the action with non-minimal coupling of the scalar filed to Ricci tensor and Ricci scalar defined by the LeviCivita connection

$$
\begin{aligned}
S=\int d^{4} x \sqrt{-g}\left[R-\left(g_{\mu \nu}+\kappa_{1} g_{\mu \nu} R+\kappa_{2} R_{\mu \nu}\right) \phi^{\mu} \phi^{\nu}-\right. \\
-2 V(\phi)],
\end{aligned}
$$

where $\phi^{\mu}=\phi_{n} u g^{\mu v}$ and two coupling constants have dimension of inverse mass squared. The Ricci scalar is defined though the Levi-Civita connection of the metric $g_{\mu \nu}$, its variation is given by

$\delta R_{\mu \nu}=\nabla^{\lambda} \nabla_{(\mu} \delta g_{\nu) \lambda}-\frac{1}{2} \square \delta g_{\mu \nu}-\frac{1}{2} g^{\lambda \rho} \nabla_{\mu} \nabla_{\nu} \delta g_{\lambda \rho}$.

Applying this to (28) and commuting some covariant derivatives one obtains the equation

$G_{\mu \nu}=T_{\mu \nu}+\kappa_{1} \Theta_{\mu \nu}^{1}+\kappa_{2} \Theta_{\mu \nu}^{2}$,

where the first terms is the minimal energy-momentum tensor, $T_{\mu \nu}=\phi_{\mu} \phi_{\nu}-g_{\mu \nu}\left(\phi_{\lambda} \phi^{\lambda} / 2-V(\phi)\right)$, while the other terms correspond to separate contributions of two nonmininmal couplings

$\Theta_{\mu \nu}^{1}=\phi_{\mu} \phi_{\nu} R-\phi_{\lambda} \phi^{\lambda} G_{\mu \nu}+\left(g_{\mu \nu} \square-\nabla_{\mu} \nabla_{\nu}\right)\left(\phi_{\lambda} \phi^{\lambda}\right)$,

$$
\begin{aligned}
\Theta_{\mu \nu}^{2}= & 2 \phi^{\alpha} \phi_{(\mu} R_{v) \alpha}-\phi^{\alpha} \nabla_{\alpha} \phi_{\mu \nu} \\
& +g_{\mu \nu}\left(\phi^{\alpha \beta} \phi_{\alpha \beta} / 2+(\square \phi)^{2} / 2+\phi^{\alpha} \nabla_{\alpha} \square \phi\right),
\end{aligned}
$$

where $\phi_{\alpha \beta}=\nabla_{\alpha} \phi_{\beta}$ and $G_{\mu \nu}$ is the Einstein tensor. Variation over $\phi$ gives the scalar equation

$\square \phi+\nabla_{\mu}\left[\nabla_{\nu} \phi\left(\kappa_{1} g^{\mu \nu} R+\kappa_{2} R^{\mu \nu}\right)\right]=0$.

Obviously, for generic values of the coupling constants $\kappa_{1}$ and $\kappa_{2}$ both the Einstein and the scalar equations contain higher derivatives of $\phi$. Collecting the third derivative terms, we find:

$\Theta_{\mu \nu}^{3}=\left(\kappa_{2}+2 \kappa_{1}\right)\left(g_{\mu \nu} \phi^{\alpha} \nabla_{\alpha} \square \phi-\phi^{\alpha} \phi_{\alpha \mu \nu}\right)$.

These terms vanish in the case

$\kappa_{2}+2 \kappa_{1}=0$,

corresponding to the Einstein tensor in the Lagrangian (28). The Ricci-terms in the scalar equation combine into the Einstein tensor as well, so, in view of the Bianchi identity $\nabla_{\mu} G^{\mu \nu}=0$, which holds in the metric theory, the Eq. (33) becomes the second order eqiation

$\left[g^{\mu \nu}+\kappa G^{\mu \nu}\right] \nabla_{\mu} \nabla_{\nu} \phi=0$.

This case belongs to the Horndesky class. 


\subsection{Palatini}

In the Palatini version, the action will read

$$
\begin{aligned}
S= & \int d^{4} x \sqrt{-g}\left[\left(\hat{R}_{\mu v}-\phi_{\mu} \phi_{v}\right) g^{\mu \nu}\right. \\
& \left.-\hat{R}_{\alpha \beta} \phi_{\mu} \phi_{v}\left(\kappa_{1} g^{\alpha \beta} g^{\mu v}+\kappa_{2} g^{\alpha \mu} g^{\beta v}\right)\right] .
\end{aligned}
$$

Similarly to the conformally coupled theory, this action includes only the symmetric part of the Ricci tensor, and it is projective invariant under (23). We therefore set torsion to zero and make variation with respect to connection according to (24). This gives the following equation for an unknown connection:

$\hat{\nabla}_{\lambda}\left(\sqrt{-g} Z^{\mu \nu}\right)=0$

$Z^{\mu \nu}=\lambda g^{\mu \nu}-\kappa_{2} \phi^{\mu} \phi^{\nu}, \quad \phi^{\mu}=\phi_{\alpha} g^{\alpha \mu}$

where we have denoted

$\lambda=\left(1-\kappa_{1} X\right), \quad X=\phi_{\alpha} \phi_{\beta} g^{\alpha \beta}$.

To solve the Eq. (38) with respect to $\hat{\Gamma}$ we would like to cast it into the form $\hat{\nabla}_{\lambda} \hat{g}_{\mu \nu}=0$ for some second metric, or to some equivalent equation. Indeed, since $Z^{\mu \nu} \sqrt{-g}$ is the tensor density we will try to introduce such a metric via an identification

$Z^{\mu \nu} \sqrt{-g}=\hat{g}^{\mu \nu} \sqrt{-\hat{g}}$

so that the determinant would be of the same metric. To proceed, we first construct the matrix $W_{\mu \nu}$, an inverse of the matrix $Z^{\mu \nu}$ :

$W_{\mu \lambda} Z^{\lambda v}=\delta_{\mu}^{v}$

It can be obtained as linear combination of $g_{\mu \nu}$ and $\phi_{\mu} \phi_{v}$ as follows

$W_{\mu \nu}=\lambda^{-1}\left(g_{\mu \nu}+\kappa_{2} \mu^{-1} \phi_{\mu} \phi_{\nu}\right)$,

where $\mu=1-\left(\kappa_{1}+\kappa_{2}\right) X$. To find the ratio of the determinants, we rewrite this in the form

$W_{\mu v}=\lambda^{-1} g_{\mu \lambda}\left(\delta_{v}^{\lambda}+M_{v}^{\lambda}\right), \quad M_{v}^{\lambda}=\kappa_{2} \lambda^{-1} \phi^{\lambda} \phi_{v}$,

where the matrix $M$ has the property $M^{2} \sim M$. For such matrices the determinant is given by

$\operatorname{det}(1+M)=1+\operatorname{tr} M$.

Then from (42) we obtain

$\operatorname{det} W=\lambda^{-4} \operatorname{det} g\left(1+\kappa_{2} X / \mu\right)=\lambda^{-3} \mu^{-1} \operatorname{det} g$.
Since the determinant of $Z^{\mu \nu}$ is inverse to det $W$, we finally find from (40) :

$\hat{g}=g \mu \lambda^{3}$,

and, using this, we obtain the second metric explicitely as

$\hat{g}_{\mu \nu}=\sqrt{\mu \lambda}\left(g_{\mu \nu}+\kappa_{2} \mu^{-1} \phi_{\mu} \phi_{\nu}\right)$.

Now the Eq. (38) becomes

$\hat{\nabla}\left(\hat{g}^{\mu \nu} \sqrt{-\hat{g}}\right)=0$

so the Palatini connection will be the Levi-Civita connection of the new metric:

$\hat{\Gamma}_{\mu \nu}^{\lambda}=\hat{g}^{\lambda \tau}\left(\partial_{\mu} \hat{g}_{\lambda \nu}+\partial_{\mu} \hat{g}_{\mu \lambda}-\partial_{\lambda} \hat{g}_{\mu \nu}\right) / 2$.

Now we turn to other equations of motion. Variation of the action (60) with respect to the metric leads to the EinsteinPalatini equation

$\lambda \hat{R}_{\mu \nu}-\phi_{\mu} \phi_{\nu}\left(1+\kappa_{1} \hat{R}\right)-2 \kappa_{2} \hat{R}_{\alpha(\mu} \phi_{\nu)} \phi^{\alpha}-g_{\mu \nu} L / 2=0$,

where the Lagrangian can be concisely presented as

$L=\hat{R}_{\mu \nu} Z^{\mu \nu}-\phi_{\mu} \phi_{\nu} g^{\mu \nu}$

Finally, a variation over $\phi$ gives rise to a scalar equation

$\partial_{\mu}\left[\sqrt{-g}\left(\phi^{\mu}+\kappa_{1} \hat{R} \phi^{\mu}+\kappa_{2} \hat{R}_{\alpha \beta} g^{\beta \mu} \phi^{\alpha}\right)\right]=0$,

which, in principle, could contain higher-derivative terms.

\subsection{Einstein frame}

So far we have obtained the second metric $\hat{g}_{\mu \nu}$ as an auxiliary one, needed to generate the Palatini connection. Note that it is related to the physical metric $g_{\mu \nu}$ by a disformal transformation (46). The inverse of $\hat{g}_{\mu \nu}$ can be read off from the Eq.(40) with account for the ratio of determiants (45):

$\hat{g}^{\mu \nu}=\mu^{-1 / 2} \lambda^{-1 / 2}\left(g^{\mu \nu}-\kappa_{2} \phi^{\mu} \phi^{\nu} / \lambda\right)$.

The functions $\lambda$ and $\mu$ depend on the initial metric through the norm of the gradient of the scalar field $X=\phi_{\mu} \phi_{\nu} g^{\mu \nu}$, so to invert the transformation one has to express $X$ through the norm with respect to the second metric $\hat{X}=\hat{g}^{\mu \nu} \phi_{\mu} \phi_{\nu}$. Contracting the Eq. (52) with $\phi_{\mu} \phi_{\nu}$ we obtain the equation

$\hat{X}=X \mu^{1 / 2} \lambda^{-3 / 2}$. 
Clearly, we have to restrict physical domain by the conditions $\mu>0, \lambda>0$. One must also avoid the critical point of the function $\hat{X}(X)$ where the derivative

$\frac{\partial \hat{X}}{\partial X}=\frac{2-X\left(2 \kappa_{1}+3 \kappa_{2}\right)}{2 \mu^{1 / 2} \lambda^{5 / 2}}$

is zero. This occurs at

$X=X_{\mathrm{cr}}=\frac{2}{2 \kappa_{1}+3 \kappa_{2}}$,

where the inverse derivative will diverge. But in the regions of monotonicity of $\hat{X}(X)$ the Eq. (53) is a cubuc equation obtained by squaring (53)

$\hat{X}^{2}\left(1-\kappa_{1} X\right)^{3}-X^{2}\left[1-\left(\kappa_{1}+\kappa_{2}\right) X\right]=0$,

whose roots can be found explicitly (for more details see [44]), so with such precautions, we can say that the transformation between two metrics is reversible.

In view of the relation

$X \sqrt{-g}=\hat{X} \mu^{-1} \sqrt{-\hat{g}}$

and the representation (50) of the Lagrangian, it is now an easy task to express it entirely in terms of the second metric:

$$
\begin{aligned}
\sqrt{-g} L & =\sqrt{-g}\left(\hat{R}_{\mu \nu} Z^{\mu \nu}-X\right) \\
& =\sqrt{-\hat{g}}\left(\hat{R}_{\mu \nu}-\mu^{-1} \phi_{\mu} \phi_{\nu}\right) \hat{g}^{\mu \nu} .
\end{aligned}
$$

We have obtained the Einstein-Hilbert term plus a modified scalar kinetic term without higher derivatives. In view of invertibility of the transformation to the Einstein frame, this means that the initial Palatini theory (60) is free of Ostrogradsky ghosts for general generic coupling constants $\kappa_{1}, \kappa_{2}$. Recall that in the metric formalism it belongs to Horndeski class only for $\kappa_{2}=-2 \kappa_{1}$.

\subsection{New Palatini kinetic coupling}

Now we see that, in the Palatini formalism, another particular relation, namely,

$\kappa_{2}=-\kappa_{1}=\kappa$

defines an exceptionally simple derivetively coupled ST theory,

$$
\begin{aligned}
S= & \int d^{4} x \sqrt{-g}\left[\left(\hat{R}_{\mu \nu}-\phi_{\mu} \phi_{v}\right) g^{\mu \nu}\right. \\
& \left.-\kappa \hat{R}_{\alpha \beta} \phi_{\mu} \phi_{\nu}\left(g^{\alpha \mu} g^{\beta \nu}-g^{\alpha \beta} g^{\mu \nu}\right)\right],
\end{aligned}
$$

in which case $\mu=1$, so our theory is disformally dual to MES is in the Einstein frame [44]:

$S_{E}=\int \sqrt{-\hat{g}}\left[R_{\mu \nu}(\hat{g})-\phi_{\mu} \phi_{\nu}\right] \hat{g}^{\mu \nu} d^{4} x$

In this dual theory the Einstein equation reads

$R_{\mu \nu}=\phi_{\mu} \phi_{\nu}$,

and the scalar obeys the covariant d'Alembert equation

$\hat{\square} \phi=0$.

Note, that for the Einstein-Hilbert lagrangian both the metric and the Palatini variations lead to the same equations, therefore, one can replace the Palatini Ricci scalar built with the Levi-Civita connection of the Einstein frame metric, by the usual metric scalar curvature

$\hat{g}^{\mu \nu} \hat{R}_{\mu \nu}(\hat{\Gamma})=R(\hat{g})$.

One can verify that Eqs. (49) and (51) are satisfied by virtue of Eqs. (62) and (63). First, we obtain that Eq. (62) implies $L=0, \hat{R}=\psi$, hence Eq. (49) holds. Using then Eq. (62) in Eq. (51), we reduce the latter to (63). For this one-parametric family of Lagrangians (note that both signs of $\kappa$ are relevant, depending on whether the $\phi_{\mu}$ is timelike or spacelike in the Einstein frame [44]).

We will be interested in the inverse disformal transformation from Einstein metric $\hat{g}_{\mu \nu}$ to Jordan metric $g_{\mu \nu}$. For this, one has to express the factor $\lambda$ through the Einstein-metric norm $\hat{X}=\phi_{\mu} \phi_{\nu} \hat{g}^{\mu \nu}$. From the Eq. (56) with account for (59) one obtains the following cubic equation for $\sqrt{\lambda}$ :

$2 z(\sqrt{\lambda / 3})^{3}+\lambda-1=0, \quad z=\frac{3 \sqrt{3}}{2} \kappa_{1} \phi_{\mu} \phi_{\nu} \hat{g}^{\mu \nu}$,

which has a real solution

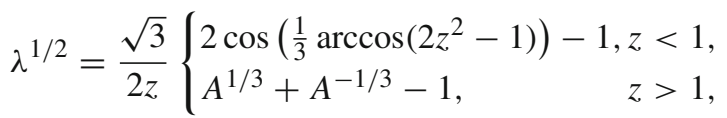

where $A=2 z \sqrt{z^{2}-1}+2 z^{2}-1$. Then the Jordan metric will read:

$g_{\mu \nu}=\hat{g}_{\mu \nu} \lambda^{-1 / 2}+\kappa_{1} \phi_{\mu} \phi_{\nu}$. 


\section{Resolution of static singularities}

\subsection{FJNW in the Einstein frame}

The minimal scalar gravity (11) has a satic spherically symmetric solution, which was first found Fisher [104] and later rediscovered by many people including Janis, Newman and Winicour [105], nowadays commonly abbreviated as FJNW:

$$
\begin{aligned}
d \hat{s}^{2}= & -\left(1-\frac{b}{r}\right)^{\gamma} d t^{2}+\left(1-\frac{b}{r}\right)^{-\gamma} d r^{2} \\
& +r^{2}\left(1-\frac{b}{r}\right)^{1-\gamma}\left(d \theta^{2}+\sin ^{2} \theta d \varphi^{2}\right), \\
\hat{\varphi} & =\frac{q}{b} \ln \left(1-\frac{b}{r}\right),
\end{aligned}
$$

where $q$ is the scalar charge and

$$
\gamma=\left(1-\frac{2 q^{2}}{b^{2}}\right)^{1 / 2}, \quad 0<\gamma<1
$$

It is asymptotically flat and has a curvature singularity at $r=b$, unless $\gamma=1$.

\subsection{Conformal theory}

Consider the case $\gamma=1 / 2$, when all irrational powers are square roots. Then $q=b \sqrt{3 / 8}$, and the Bekenstein's transformation reads:

$$
\begin{aligned}
\phi & =\sqrt{6} \tanh (\hat{\varphi} / \sqrt{6})=\frac{\sqrt{1-b / r}-1}{\sqrt{1-b / r}+1}, \\
d s^{2} & =\left(1-\phi^{2} / 6\right)^{-1} d \hat{s}^{2} .
\end{aligned}
$$

Now perform the coordinate transformation:

$1-\frac{b}{r}=\left(1-\frac{b}{2 \rho}\right)^{2}$

In terms of the new coordinates, the solution takes the BBMB form [73]:

$$
\begin{aligned}
& \phi=\frac{\sqrt{6} m}{\rho-m}, \quad m=\frac{b}{4} \\
& d s^{2}=-\left(1-\frac{m}{\rho}\right)^{2} d t^{2}+\left(1-\frac{m}{\rho}\right)^{-2} d \rho^{2}+\rho^{2} d \Omega .
\end{aligned}
$$

The metric conincides with the Reissner-Nordstrom extremal solution, while the scalar field diverges on the horizion. As was shown by Bekenstein [75], the singularity is unseen by a particle interacting with this scalar, so the solution as a whole can be regarded as a legitimate black hole. Thus, a naked singularity solution of MES was converted into a black hole solution in the Jordan frame of the $R \phi^{2}$ theory. But the singularity inside the horizon still remained. As was noted in [106], the FJNW singularity $r=b$ is mapped onto a regular surface $\rho=2 m$, while the horizon is at $\rho=m$; this was be interpreted in [106] as conformal continuation of the MES solution through the singularity. More recently there was a renewed interest to construct ST solutions starting with new MES solutions including time-dependent ones [107-109].

\subsection{New kinetic theory}

Now transform FJNW to the Jordan frame of the new kinetically coupled theory (60). In the static case, interesting solutions arise for $\kappa_{1}=-\kappa_{2}>0$, so here we denote $\kappa=\kappa_{1}$ (or invert the sign of $\kappa$ in (60) taking $\kappa$ positive again). The disformal transformation (67) generates now the new metric according to the rules

$g_{t t}=\frac{\hat{g}_{t t}}{\lambda^{1 / 2}}, \quad g_{r r}=\frac{\hat{g}_{r r}}{\lambda^{3 / 2},} \quad g_{\theta \theta}=\frac{\hat{g}_{r \theta, \theta}}{\lambda^{1 / 2}}$,

where the factor $\lambda$ is obtained using the Eq. (67):

$$
\begin{aligned}
\lambda^{-3 / 2}\left(1-\frac{b}{r}\right)^{-\gamma} & \\
= & \frac{2 x}{3 \sqrt{3}}+\frac{1}{\sqrt{3}} \begin{cases}2 w \cos \left[\frac{1}{3} \arccos (x / w)\right], & x<w, \\
w^{2 / 3} B+w^{4 / 3} B^{-1}, & x>w,\end{cases}
\end{aligned}
$$

with

$B=\left(x+\sqrt{x^{2}-w^{2}}\right)^{1 / 3}, \quad x=\frac{3 \sqrt{3} \kappa q^{2}}{2 r^{2}(r-b)^{2}}$.

For large $r$ the variable $x \sim 1 / r^{4}$, so $\lambda=1+O\left(r^{-4}\right)$ and the solution remains asymptotically flat:

$g_{t t} \sim-1+\frac{\gamma b}{r}, \quad g_{r r} \sim 1-\frac{\gamma b}{r}, \quad g_{\theta \theta} \sim r^{2}$.

Near the MES singularity $r=b$ one can expand in terms of $\xi=(r-b) / b$, denoting $\kappa q^{2} / b^{4}=v^{3}$ :

$$
\begin{aligned}
v^{-1} d s^{2}= & -\xi^{2(2 \gamma-1) / 3} d t^{2}+(v b / \xi)^{2} d \xi^{2} \\
& +b^{2} \xi^{(1-2 \gamma) / 3}\left(d \theta^{2}+\sin ^{2} \theta d \varphi^{2}\right)
\end{aligned}
$$

In the case $\gamma=1 / 2$, passing to a new variable $z=$ $v b \ln \xi,-\infty<z<\infty$, one obtains

$v^{-1} d s^{2}=-d t^{2}+d z^{2}+b^{2}\left(d \theta^{2}+\sin ^{2} \theta d \varphi^{2}\right)$.

This metric represent the product of a two-dimensional Minkowsky space and a two-sphere. Note that the scalar field 
is not transformed and remains singular at $r=b$. But the disformal transformation appropriately subtracts the divergence from the metric. Desingularization is stronger in this case, since the resulting metric is geodesically complete and non-singular.

\section{Cosmology}

\subsection{MES cosmology with $\Lambda$}

Consider homogeneous and isotropic cosmologies in Einstein's theory minimally coupled to scalar in presence of the cosmological constant. We assume the metric parametrization:

$d \hat{s}^{2}=-\hat{N}^{2} d t^{2}+\hat{a}^{2} d l_{k}^{2}, \quad d l_{k}^{2}=d \chi^{2}+f_{k} d \Omega^{2}$,

where $k=0, \pm 1$, with $f_{1}=\sin ^{2} \chi, f_{0}=\chi^{2}, f_{-1}=$ $\cosh ^{2} \chi$ for spatially closed, flat and open universes respectively, and the functions $\hat{N}, \hat{a}$ depend only on $t$. Note that our time $t$ and the three-space coordinates are dimensionless, while the functions $\hat{N}, \hat{a}$ have the dimension of length. We obtain the following relevant components of the Ricci tensor:

$\hat{R}_{t t}=\frac{3 \dot{\hat{N}} \dot{\hat{a}}}{\hat{N} \hat{a}}-\frac{3 \ddot{\hat{a}}}{\hat{a}}$,

$\hat{R}_{\chi \chi}=\frac{\hat{a} \ddot{\hat{a}}}{\hat{N}^{2}}-\frac{\hat{a} \dot{\hat{N}} \dot{\hat{a}}}{\hat{N}^{3}}+\frac{2 \dot{\hat{a}}^{2}}{\hat{N}^{2}}+2 k$.

The Einstein equations read:

$\hat{R}_{\mu \nu}=\Lambda \hat{g}_{\mu \nu}+\partial_{\mu} \hat{\varphi} \partial_{\nu} \hat{\varphi}$.

The equation for $\hat{R}_{\chi \chi}$ does not contain the scalar field and admits the first integral:

$\frac{\hat{a}^{4} \dot{\hat{a}}^{2}}{\hat{N}^{2}}+k \hat{a}^{4}+\frac{1}{3} \Lambda \hat{a}^{6}=\left(2 a_{0}\right)^{4}$,

using which we find:

$$
\begin{aligned}
& \hat{N}^{2}=\frac{\hat{a}^{4} \dot{\hat{a}}^{2}}{\left(2 a_{0}\right)^{4}-k \hat{a}^{4}+\Lambda \hat{a}^{6} / 3}, \\
& \dot{\hat{\varphi}}^{2}=\frac{\dot{\hat{a}}^{2}}{\hat{a}^{2}\left(\left(2 a_{0}\right)^{4}-3 k \hat{a}^{4}+\Lambda \hat{a}^{6} / 3\right)} .
\end{aligned}
$$

We still have freedom to fix the gauge, the convenient one being $\hat{a}=2 a_{0} t$. Then

$$
\begin{aligned}
& \hat{N}^{2}=\frac{\left(2 a_{0}\right)^{2} t^{4}}{1-k t^{4}+4\left(a_{0}\right)^{2} \Lambda t^{6} / 3} \\
& \dot{\hat{\varphi}}^{2}=\frac{6}{t^{2}\left(1-3 k t^{4}+4\left(a_{0}\right)^{2} \Lambda t^{6} / 3\right)}
\end{aligned}
$$

Note that near the cosmological singularity, both the spatial curvature terms and the $\Lambda$-terms are negligible.

\subsection{Minkowsky start of the universe in $R \phi^{2}$}

Performing the Bekenstein's transformations in the case $\Lambda=0, k=0$, one obtains the following exact cosmological solution of the theory (17):

$$
\begin{aligned}
\phi / \sqrt{6} & =\tanh (\hat{\varphi} / \sqrt{6})=\frac{t^{2}-1}{t^{2}+1} \\
d s^{2} & =\left(1-\phi^{2} / 6\right)^{-1} d \hat{s}^{2}=\frac{\left(t^{2}+1\right)^{2}}{4 t^{2}} d \hat{s}^{2} \\
& =\left(t^{2}+1\right)^{2}\left[-\left(4 a_{0} t\right)^{2} d t^{2}+a_{0}^{2} d l_{0}^{2}\right] .
\end{aligned}
$$

In terms of the synchronous time,

$$
\tau=a_{0} t^{2}\left(t^{2}+2\right), \quad \text { or } \quad t^{2}=\sqrt{1+\tau / a_{0}}-1,
$$

we obtain:

$d s^{2}=-d \tau^{2}+a^{2} d l_{0}^{2}, \quad a=a_{0}\left(t^{2}+1\right)=a_{0} \sqrt{1+\tau / a_{0}}$.

Thus the univers starts from the Minkowsky stage. The Hubble parameter and its derivative are:

$H=\frac{1}{a} \frac{d a}{d \tau}=\frac{1}{2\left(a_{0}+\tau\right)}, \quad \dot{H}=\frac{d H}{d \tau}=-2 H^{2}$.

The universe is always decelerating.

When $k= \pm 1, \Lambda \neq 0$, the very beginning of the expansion obeys the same law.

\subsection{New kinetic theory: Genesis}

Now transform the MES cosmological solution into the Jordan frame of the new Palatini kinetically coupled theory (60). In this case, the relevant sign of the coupling constant $\kappa$ is positive. We will be interested in the behavior of the scale factor near the singularity of the MES solution. Since in this case both the cosmological constant and the curvature term 
are negligible, we start with $k=0, \Lambda=0$, choosing the synchronous gauge:

$d \hat{s}^{2}=-d t^{2}+\hat{a}^{2} d l_{0}^{2}$

where

$\hat{a}=a_{0} t^{1 / 3}, \quad \phi=\sqrt{2} \ln t / \sqrt{3}$,

as was found by Zel'dovich in 1972 for the stiff-matter [110, 111]. Obviously, this metric is singular at $t=0$ and describes a decelerating expansion.

Now we transform the metric to the Jordan frame of the new kinetic theory. From (67) we obtain an algebraic equation for $N$ :

$$
(N-2 z /(3 \sqrt{3}))^{3}=N^{2}, \quad z=\kappa \sqrt{3} / t^{2} .
$$

Its real solution is smooth, though in the form below it looks piecewise:

$N^{2}=\frac{2 z}{3 \sqrt{3}}+\frac{1}{\sqrt{3}} \begin{cases}2 \cos \left(\frac{1}{3} \arccos (x)\right), & z<1, \\ A^{1 / 3}+A^{-1 / 3}, & z>1,\end{cases}$

where $A=\left(z+\sqrt{z^{2}-1}\right)^{1 / 3}$. For large $z$ (small $t$ ) one has:

$N^{2}=2 z / 3 \sqrt{3}+(2 z)^{\frac{1}{3}} / \sqrt{3}+(4 / z)^{\frac{1}{3}} /(2 \sqrt{3})+\ldots$,

or, in terms of time,

$N^{2}=(\alpha t)^{-2}\left(1+(\alpha t)^{4 / 3}\right), \quad \alpha=\left(\frac{3}{2 \kappa}\right)^{1 / 2}$.

For the scale factor we obtain $a^{2}=\hat{a}^{2} N^{2 / 3}$, so keeping the first next to leading terms, the metric will read:

$$
\begin{aligned}
d s^{2}= & -(\alpha t)^{-2}\left(1+(\alpha t)^{4 / 3}\right) d t^{2} \\
& +a_{1}^{2}\left(1+(\alpha t)^{4 / 3} / 3\right) d l_{0}^{2}, \quad a_{1}=a_{0} \alpha^{-1 / 3} .
\end{aligned}
$$

We need to go to the synchronous time $t \rightarrow \tau(t)$ solving the equation $N d t=d \tau$. For small $t$, keeping the leading term in (99), one finds:

$d t / d \tau=\alpha t \Longrightarrow t=\mathrm{e}^{\alpha \tau}$,

so that $t \rightarrow 0$ corresponds $\tau \rightarrow-\infty$.

Now compute the Hubble parameter differentiating with respect to the synchronous time in the vicinity of $t=0$ :

$H=\frac{1}{a} \frac{d a}{d t} \frac{d t}{d \tau}=\frac{2 \alpha}{9}(\alpha t)^{4 / 3}$.
Its derivative in the leading order reads

$\dot{H}=\frac{d H}{d \tau}=\frac{8 \alpha^{2}}{27}(\alpha t)^{4 / 3}$,

and satifies the strong NEC violation contidion: the ratio

$\frac{\dot{H}}{H^{2}}=\frac{6}{(\alpha t)^{4 / 3}}=\frac{6}{\alpha^{4 / 3}} \mathrm{e}^{-4 \alpha \tau / 3}$

diverges exponentially as $\tau \rightarrow-\infty$. Such a behavior is typical for the Genesis scenario [66,67]. The universe starts from the Minkowsky stage with positive acceleration. Thus, the NEC violation is more pronounced in the new kinetic theory than in the conformal theory. Similar behavior near the singularity was observed in [112] in a different setting. Desingularization by field redefinition was recently discussed in [113].

\section{Conclusions}

Our goal was to draw attention to successive duaities in the non-minimal scalar-tensor theories without matter that arise when two or more theories coincide in their respective Einstein frames, into which they can be transformed by means of invertible mappings. By the group property of reversible mappings, these theories are directly related by an overall invertible transformation, thus they are dual to each other. If one of them is free from Ostrogradski ghosts, the partner theory will also share this property. As we have seen, successive dualities may relate theories looking quite differently, such as $R \phi^{2}$ theory without derivatives and kinetically coupled theories. Combining them into one class can be useful for understanding the landscape of a complete set of ST theories.

Such successive dualities are especially useful if the Einstein frame theory is simply the minimally coupled Einsteinscalar theory. In this case using the known exact solutions of the latter as seeds, one can construct exact solutions of the non-minimal ST theories which are quite rare. According to this construction, they can be regarded as two Jordan frames of the unique underlying theory. Although the addition of matter can destroy this symmetry, some properties, such as behavior near the singularities of the Einstein theoiry, are often not affected by matter, therefore successive dualities can be useful for comparing desingularization features due to NEC violation in these theories. We have found that desingularization of the Fisher static solution of the MES theory is stronger in the kinetic frame where it looks as globally regular solution, while in the conformal frame it is globally a black hole. The singularity of the homogeneous and isotropic cosmoilogical solution of MES is absent in both conformal 
and kinetic frames, but in the latter case violating of NEC is more pronounced, leading to Genesis-type behavior.

It would be interesting to look for other derivatively coupled theories admitting the MES representaion. Also, class of successive dualities can be extended taking MES with potentials, which also allow for exact solutions. These will generate non-minimal STs which will be ghost-free as well, though generically they will not have such a simple form in their Jordan frames as in our examples here.

Acknowledgements The author is grateful to Gérard Clément for careful reading of the manuscript and valuable comments. He also thanks Evgeny Babichev, Andrei Barvinsky, Salvatore Cappozziello, Jose Beltrán Jiménez, Sergei Sushkov, Michael Volkov and especially Kirill Bronnikov for valuable remarks and discussion. The work was supported by the Russian Foundation for Basic Research grant 17-0201299a. The networking support by the COST Action CA16104 is acknowledged.

Data Availability Statement This manuscript has associated data in a data repository. [Authors' comment: All data included in this manuscript are available upon request by contacting with the author.]

Open Access This article is licensed under a Creative Commons Attribution 4.0 International License, which permits use, sharing, adaptation, distribution and reproduction in any medium or format, as long as you give appropriate credit to the original author(s) and the source, provide a link to the Creative Commons licence, and indicate if changes were made. The images or other third party material in this article are included in the article's Creative Commons licence, unless indicated otherwise in a credit line to the material. If material is not included in the article's Creative Commons licence and your intended use is not permitted by statutory regulation or exceeds the permitted use, you will need to obtain permission directly from the copyright holder. To view a copy of this licence, visit http://creativecomm ons.org/licenses/by/4.0/.

Funded by SCOAP ${ }^{3}$.

\section{References}

1. S. Capozziello, M. De Laurentis, Extended Theories of Gravity. Phys. Rep. 509, 167 (2011)

2. S. Nojiri, S.D. Odintsov, V.K. Oikonomou, Modified Gravity Theories on a Nutshell: Inflation, Bounce and Late-time Evolution. Phys. Rep. 692, 1 (2017)

3. L. Heisenberg, "A systematic approach to generalisations of General Relativity and their cosmological implications," arXiv: 1807.01725 [gr-qc]

4. D. Langlois, Dark energy and modified gravity in degenerate higher-order scalar-tensor (DHOST) theories: A review. Int. J. Mod. Phys. D 28(05), 1942006 (2019). arXiv:1811.06271 [gr-qc]

5. L. Barack et al., Black holes, gravitational waves and fundamental physics: a roadmap. Class. Quant. Grav. 36(14), 143001 (2019). arXiv: 1806.05195 [gr-qc]

6. T. Kobayashi, Horndeski theory and beyond: a review. Rept. Prog. Phys. 82(8), 086901 (2019). arXiv:1901.07183 [gr-qc]

7. E.E. Flanagan, R.M. Wald, Does back reaction enforce the averaged null energy condition in semiclassical gravity? Phys. Rev. D 54, 6233 (1996). arXiv:gr-qc/9602052
8. S. Mandal, Revisiting Laws of Black Hole Mechanics and Violation of Null Energy Condition. J. High Energy Phys. Gravit. Cosmol. 5, 82-111 (2019)

9. V. A. Rubakov, "The Null Energy Condition and its violation," Phys. Usp. 57, 128 (2014) [Usp. Fiz. Nauk 184(2), 137 (2014)] arXiv: 1401.4024 [hep-th]

10. N.A. Chernikov, E.A. Tagirov, "Quantum theory of scalar fields in de Sitter space-time,". Ann. Inst. H. Poincare Phys. Theor. A 9, 109 (1968)

11. C.G. Callan Jr., S.R. Coleman, R. Jackiw, A New improved energy - momentum tensor. Ann. Phys. 59, 42 (1970)

12. L. Parker, Conformal energy-momentum tensor in riemannian space-time. Phys. Rev. D 7, 976 (1973)

13. S. Bellucci, V. Faraoni, Energy conditions and classical scalar fields. Nucl. Phys. B 640, 453 (2002). arXiv:hep-th/0106168

14. J.D. Bekenstein, Nonsingular General Relativistic Cosmologies. Phys. Rev. D 11, 2072 (1975)

15. S.S. Bayin, F.I. Cooperstock, V. Faraoni, A Singularity - free cosmological model with a conformally coupled scalar field. Astrophys. J. 428, 439 (1994). arXiv:astro-ph/9402033

16. A. Saa, E. Gunzig, L. Brenig, V. Faraoni, T.M. Rocha Filho, A. Figueiredo, Superinflation, quintessence, and the avoidance of the initial singularity. Int. J. Theor. Phys. 40, 2295 (2001). arXiv:gr-qc/0012105

17. E. Gunzig, A. Saa, L. Brenig, V. Faraoni, T.M. Rocha Filho, A. Figueiredo, Superinflation, quintessence, and nonsingular cosmologies. Phys. Rev. D 63, 067301 (2001). arXiv:gr-qc/0012085

18. L.R. Abramo, L. Brenig, E. Gunzig, A. Saa, On the singularities of gravity in the presence of nonminimally coupled scalar fields. Phys. Rev. D 67, 027301 (2003). [arXiv:gr-qc/0210069]

19. J.L. Cervantes-Cota, H. Dehnen, Induced gravity inflation in the standard model of particle physics. Nucl. Phys. B 442, 391 (1995). arXiv:astro-ph/9505069

20. F.L. Bezrukov, M. Shaposhnikov, The Standard Model Higgs boson as the inflaton. Phys. Lett. B 659, 703 (2008). arXiv:0710.3755 [hep-th]

21. F. Bauer, D.A. Demir, Inflation with Non-Minimal Coupling: Metric versus Palatini Formulations. Phys. Lett. B 665, 222 (2008). arXiv:0803.2664 [hep-ph]

22. C.P. Burgess, H.M. Lee, M. Trott, Power-counting and the Validity of the Classical Approximation During Inflation. JHEP 0909, 103 (2009). arXiv:0902.4465 [hep-ph]

23. C. Germani, A. Kehagias, New Model of Inflation with Nonminimal Derivative Coupling of Standard Model Higgs Boson to Gravity. Phys. Rev. Lett. 105, 011302 (2010). arXiv:1003.2635 [hep-ph]

24. S. Sato, Ki Maeda, Hybrid Higgs Inflation: The Use of Disformal Transformation. Phys. Rev. D 97(8), 083512 (2018). arXiv:1712.04237 [gr-qc]

25. L. Amendola, Cosmology with nonminimal derivative couplings. Phys. Lett. B 301, 175 (1993)

26. S. Capozziello, G. Lambiase, Nonminimal derivative coupling and the recovering of cosmological constant. Gen. Relativ. Gravit. 31, 1005 (1999). arXiv:gr-qc/9901051

27. S. Capozziello, G. Lambiase, H.J. Schmidt, Nonminimal derivative couplings and inflation in generalized theories of gravity. Ann. Phys. 9, 39 (2000)

28. S.V. Sushkov, Exact cosmological solutions with nonminimal derivative coupling. Phys. Rev. D 80, 103505 (2009)

29. S.V. Sushkov, Exact cosmological solutions with nonminimal derivative coupling. Phys. Rev. D 85, 123520 (2012)

30. L.N. Granda, W. Cardona, General Non-minimal Kinetic coupling to gravity. JCAP 1007, 021 (2010)

31. L.N. Granda, D.F. Jimenez, Dynamical analysis for a scalar-tensor model with kinetic and nonminimal couplings. Int. J. Mod. Phys. D 27(03), 1850030 (2017). arXiv:1710.07273 [gr-qc] 
32. L.N. Granda, D.F. Jimenez, Slow-Roll Inflation in Scalar-Tensor Models. JCAP 1909, 007 (2019). arXiv:1905.08349 [gr-qc]

33. G.W. Horndeski, Second-order scalar-tensor field equations in a four-dimensional space. Int. J. Theor. Phys. 10, 363-384 (1974)

34. C. Deffayet, X. Gao, D.A. Steer, G. Zahariade, From k-essence to generalized Galileons. Phys. Rev. D 84, 064039 (2011)

35. C. Charmousis, E.J. Copeland, A. Padilla, P.M. Saffin, General second order scalar-tensor theory, self tuning, and the Fab Four. Phys. Rev. Lett. 108, 051101 (2012)

36. M. Zumalacarregui, J. Garcia-Bellido, Transforming gravity: from derivative couplings to matter to second-order scalar-tensor theories beyond the Horndeski Lagrangian. Phys. Rev. D 89, 064046 (2014)

37. J. Gleyzes, D. Langlois, F. Piazza, F. Vernizzi, Exploring gravitational theories beyond Horndeski. JCAP 1502, 018 (2015)

38. D. Langlois, K. Noui, Degenerate higher derivative theories beyond Horndeski: evading the Ostrogradski instability. JCAP 1602, 034 (2016). arXiv: 1510.06930 [gr-qc]

39. T.P. Sotiriou, Unification of inflation and cosmic acceleration in the Palatini formalism. Phys. Rev. D 73, 063515 (2006)

40. T. Harko, T.S. Koivisto, F.S.N. Lobo, Palatini formulation of modified gravity with a nonminimal curvature-matter coupling. Mod. Phys. Lett. A 26, 1467 (2011)

41. G.J. Olmo, Palatini Approach to Modified Gravity: $\mathrm{f}(\mathrm{R})$ Theories and Beyond. Int. J. Mod. Phys. D 20, 413 (2011)

42. X. Luo, P. Wu, H. Yu, Non-minimal derivatively coupled quintessence in the Palatini formalism. Astrophys. Space Sci. 350(2), 831 (2014)

43. E. Davydov, Comparing metric and Palatini approaches to vector Horndeski theory. Int. J. Mod. Phys. D 27(04), 1850038 (2017)

44. D. Gal'tsov, S. Zhidkova, Ghost-free Palatini derivative scalartensor theory: Desingularization and the speed test. Phys. Lett. B 790, 453 (2019). arXiv:1808.00492 [hep-th]

45. G.J. Olmo, H. Sanchis-Alepuz, S. Tripathi, Dynamical Aspects of Generalized Palatini Theories of Gravity. Phys. Rev. D 80, 024013 (2009)

46. N. Kaewkhao, B. Gumjudpai, Cosmology of non-minimal derivative coupling to gravity in Palatini formalism and its chaotic inflation. Phys. Dark Univ. 20, 20 (2018)

47. R. Jinno, M. Kubota, K. y. Oda, S. C. Park, "Higgs inflation in metric and Palatini formalisms: Required suppression of higher dimensional operators," arXiv:1904.05699 [hep-ph]

48. T. Helpin, M. S. Volkov, "Varying the Horndeski Lagrangian within the Palatini approach," arXiv:1906.07607 [hep-th]

49. S. Capozziello, T. Harko, T.S. Koivisto, F.S.N. Lobo, G.J. Olmo, Hybrid metric-Palatini gravity. Universe 1(2), 199 (2015)

50. S. Capozziello, F. Darabi, D. Vernieri, Mod. Phys. Lett. A 26, 65 (2011)

51. I. Quiros, R. De Arcia, I. Delgado Gaspar, R. Garcia-Salcedo, T. Gonzalez, F. A. Horta-Rangel, "An issue with the classification of the most general scalar-tensor theories," arXiv:1905.08177 [grqc]

52. J.D. Bekenstein, The Relation between physical and gravitational geometry. Phys. Rev. D 48, 3641 (1993)

53. D. Bettoni, S. Liberati, Disformal invariance of second order scalar-tensor theories: Framing the Horndeski action. Phys. Rev. D 88, 084020 (2013)

54. G. Domenech, A. Naruko, M. Sasaki, Cosmological disformal invariance. JCAP 1510(10), 067 (2015)

55. J. Sakstein, S. Verner, Disformal Gravity Theories: A Jordan Frame Analysis. Phys. Rev. D 92(12), 123005 (2015)

56. JBen Achour, D. Langlois, K. Noui, Degenerate higher order scalar-tensor theories beyond Horndeski and disformal transformations. Phys. Rev. D 93(12), 124005 (2016)
57. J. Ben Achour, H. Liu, S. Mukohyama, "Hairy black holes in DHOST theories: Exploring disformal transformation as a solution-generating method," arXiv:1910.11017 [gr-qc]

58. Q. Exirifard, M.M. Sheikh-Jabbari, Phys. Lett. B 661, 158 (2008)

59. N. Deruelle, J. Rua, JCAP 1409, 002 (2014)

60. S. Tsujikawa, JCAP 1504(04), 043 (2015)

61. F. Arroja, N. Bartolo, P. Karmakar, S. Matarrese, JCAP 1509, 051 (2015)

62. G. Domenech, S. Mukohyama, R. Namba, A. Naruko, R. Saitou, Y. Watanabe, Phys. Rev. D 92(8), 084027 (2015)

63. C. de Rham, A. Matas, JCAP 1606(06), 041 (2016)

64. K. Takahashi, H. Motohashi, T. Suyama, T. Kobayashi, Phys. Rev. D 95(8), 084053 (2017)

65. E. Babichev, K. Izumi, N. Tanahashi, M. Yamaguchi, "Invertible field transformations with derivatives: necessary and sufficient conditions," arXiv:1907.12333 [hep-th]

66. P. Creminelli, A. Nicolis, E. Trincherini, Galilean Genesis: An Alternative to inflation. JCAP 1011, 021 (2010). arXiv:1007.0027 [hep-th]

67. S. Mironov, V. Rubakov, V. Volkova, Genesis with general relativity asymptotics in beyond Horndeski theory. Phys. Rev. D 100(8), 083521 (2019). arXiv:1905.06249 [hep-th]

68. C.J. Fewster, G.J. Galloway, Singularity theorems from weakened energy conditions. Class. Quantum Gravity 28, 125009 (2011). arXiv: 1012.6038 [gr-qc]

69. P.J. Brown, C.J. Fewster, E.A. Kontou, A singularity theorem for Einstein-Klein-Gordon theory. Gen. Relativ. Gravit. 50(10), 121 (2018). arXiv:1803.11094 [gr-qc]

70. C. J. Fewster, E. A. Kontou, "A new derivation of singularity theorems with weakened energy hypotheses," arXiv:1907.13604 [gr-qc]

71. R.V. Wagoner, Scalar tensor theory and gravitational waves. Phys. Rev. D 1, 3209 (1970)

72. K.A. Bronnikov, Scalar-tensor theory and scalar charge. Acta Phys. Polon. B 4, 251 (1973)

73. J.D. Bekenstein, Exact solutions of Einstein conformal scalar equations. Ann. Phys. 82, 535 (1974)

74. N.M. Bocharova, K.A. Bronnikov, V.N. Melnikov, "On one exact solution of the system of Einstein equations and massless scalar field,”. Vestn. Mosk. Univ. Ser. III Fiz. Astron. 25(6), 706-709 (1970)

75. J.D. Bekenstein, Black Holes with Scalar Charge. Ann. Phys. 91, 75 (1975)

76. S. Deser, Improvement Versus Stability in Gravity Scalar Coupling. Phys. Lett. 134B, 419 (1984)

77. T. Futamase, Ki Maeda, Chaotic Inflationary Scenario in Models Having Nonminimal Coupling With Curvature. Phys. Rev. D 39, 399 (1989)

78. H.J. Schmidt, The Equivalence of Conformally and Minimally Coupled Scalar Fields in Einstein's Theory of Gravity. Phys. Lett. B 214, 519 (1988)

79. R. Fakir, W.G. Unruh, Improvement on cosmological chaotic inflation through nonminimal coupling. Phys. Rev. D 41, 1783 (1990)

80. N. Makino, M. Sasaki, The Density perturbation in the chaotic inflation with nonminimal coupling. Prog. Theor. Phys. 86, 103 (1991)

81. K i Maeda, Towards the Einstein-Hilbert Action via Conformal Transformation. Phys. Rev. D 39, 3159 (1989)

82. D.V. Galtsov, B.C. Xanthopoulos, A Generating technique for Einstein gravity conformally coupled to a scalar field with Higgs potential. J. Math. Phys. 33, 273 (1992)

83. J.P. Abreu, P. Crawford, J.P. Mimoso, Exact conformal scalar field cosmologies. Class. Quantum Gravity 11, 1919 (1994). arXiv:gr-qc/9401024 
84. B.C. Xanthopoulos, T.E. Dialynas, Einstein gravity coupled to a massless conformal scalar field in arbitrary space-time dimensions. J. Math. Phys. 33, 1463 (1992)

85. G. Magnano, L.M. Sokolowski, On physical equivalence between nonlinear gravity theories and a general relativistic selfgravitating scalar field. Phys. Rev. D 50, 5039 (1994). arXiv:gr-qc/9312008

86. D. Wands, Extended gravity theories and the EinsteinHilbert action. Class. Quantum Gravity 11, 269 (1994). arXiv:gr-qc/9307034

87. E.E. Flanagan, The Conformal frame freedom in theories of gravitation. Class. Quantum Gravity 21, 3817 (2004). arXiv:gr-qc/0403063

88. T.P. Sotiriou, V. Faraoni, S. Liberati, Theory of gravitation theories: A No-progress report. Int. J. Mod. Phys. D 17, 399 (2008). arXiv:0707.2748 [gr-qc]

89. V. Faraoni, E. Gunzig, Einstein frame or Jordan frame? Int. J. Theor. Phys. 38, 217 (1999). arXiv:astro-ph/9910176

90. V. Faraoni, Nonminimal coupling of the scalar field and inflation. Phys. Rev. D 53, 6813 (1996). arXiv:astro-ph/9602111

91. V. Faraoni, E. Gunzig, P. Nardone, Conformal transformations in classical gravitational theories and in cosmology. Fund. Cosmic Phys. 20, 121 (1999). arXiv:gr-qc/9811047

92. G. Allemandi, M. Capone, S. Capozziello, M. Francaviglia, Conformal aspects of Palatini approach in extended theories of gravity. Gen. Relativ. Gravit. 38, 33 (2006). arXiv:hep-th/0409198

93. S. Chakraborty, S. SenGupta, Eur. Phys. J. C 76(10), 552 (2016). arXiv:1604.05301 [gr-qc]

94. S. Capozziello, R. de Ritis, A.A. Marino, Some aspects of the cosmological conformal equivalence between 'Jordan frame' and 'Einstein frame. Class. Quantum Gravity 14, 3243 (1997)

95. M. Tsamparlis, A. Paliathanasis, S. Basilakos, S. Capozziello, Conformally related metrics and Lagrangians and their physical interpretation in cosmology. Gen. Relativ. Gravit. 45, 2003 (2013)

96. A.Y. Kamenshchik, C.F. Steinwachs, Phys. Rev. D 91(8), 084033 (2015). arXiv:1408.5769 [gr-qc]

97. M.S. Ruf, C.F. Steinwachs, Phys. Rev. D 97(4), 044050 (2018). arXiv:1711.07486 [gr-qc]

98. N. Sk, A.K. Sanyal, On the equivalence between different canonical forms of F(R) theory of gravity. Int. J. Mod. Phys. D 27(08), 1850085 (2018). arXiv:1708.02624 [gr-qc]]

99. N. Ohta, "Quantum equivalence of $f(R)$ gravity and scalartensor theories in the Jordan and Einstein frames," PTEP 2018(3), 033B02 (2018) arXiv:1712.05175 [hep-th]
100. K. Shimada, K. Aoki, Ki Maeda, Metric-affine Gravity and Inflation. Phys. Rev. D 99(10), 104020 (2019). arXiv:1812.03420 [grqc]

101. A.N. Bernal, B. Janssen, A. Jimenez-Cano, J.A. Orejuela, M. Sanchez, P. Sanchez-Moreno, On the (non-)uniqueness of the Levi-Civita solution in the Einstein-Hilbert-Palatini formalism. Phys. Lett. B 768, 280 (2017). arXiv:1606.08756 [gr-qc]

102. V. I. Afonso, C. Bejarano, J. Beltran Jimenez, G. J. Olmo, E. Orazi, "The trivial role of torsion in projective invariant theories of gravity with non-minimally coupled matter fields," Class. Quant. Grav. 34(23), 235003 (2017)

103. JBeltran Jimenez, A. Delhom, Ghosts in metric-affine higher order curvature gravity. Eur. Phys. J. C 79(8), 656 (2019). arXiv: 1901.08988 [gr-qc]

104. I.Z. Fisher, "Scalar mesostatic field with regard for gravitational effects" Zh. Exp. Teor. Fiz., v. 18, 636-640 (1948), translated in arXiv: gr-qc/9911008

105. A.I. Janis, E.T. Newman, J. Winicour, Reality of the Schwarzschild singularity. Phys. Rev. Lett. 20, 878 (1968)

106. K.A. Bronnikov, Scalar tensor gravity and conformal continuations. J. Math. Phys. 43, 6096 (2002). arXiv:gr-qc/0204001

107. J. Sultana, Generating time dependent conformally coupled Einstein-scalar solutions. Gen. Relativ. Gravit. 47(7), 73 (2015)

108. A. Banijamali, B. Fazlpour, V. Faraoni, Wyman's other scalar field solution, Sultana's generalization, and their Brans-Dicke and R2 relatives. Phys. Rev. D 100(6), 064017 (2019). arXiv:1905.07023 [gr-qc]

109. M. Astorino, Stationary axisymmetric spacetimes with a conformally coupled scalar field. Phys. Rev. D 91, 064066 (2015). arXiv:1412.3539 [gr-qc]

110. Ya. B. Zeldovich, "A Hypothesis, unifying the structure and the entropy of the Universe," Mon. Not. Roy. astr. Soc. 160, Short communications, 1p (1972)

111. P.H. Chavanis, Cosmology with a stiff matter era. Phys. Rev. D 92(10), 103004 (2015)

112. N. Kaloper, Disformal inflation. Phys. Lett. B 583, 1 (2004)

113. A. Naruko, C. M. Yoo, M. Sasaki, "Possible resolution of a spacetime singularity with field transformations," arXiv:1903.10763 [gr-qc] 